\title{
BMJ Open Exploring the experiences and perspectives of substitute decision- makers involved in decisions about deceased organ donation: a qualitative study protocol
}

\author{
Jacob Crawshaw (D) , , Justin Presseau, ${ }^{1,2}$ Zack van Allen, ${ }^{1,2}$ \\ Livia Pinheiro Carvalho, ${ }^{3}$ Kim Jordison, ${ }^{4}$ Shane English, ${ }^{1,5}$ Dean A Fergusson, ${ }^{1,2}$ \\ Francois Lauzier, ${ }^{6,7}$ Alexis F Turgeon (D) , ${ }^{6,7}$ Aimee J Sarti, ${ }^{8}$ Claudio Martin, ${ }^{9}$ \\ Frédérick D'Aragon, ${ }^{10,11}$ Alvin Ho-ting Li, ${ }^{1}$ Greg Knoll, ${ }^{12}$ Ian Ball, ${ }^{13}$ Jamie Brehaut, ${ }^{1}$ \\ Karen E A Burns, ${ }^{14}$ Marie-Chantal Fortin, ${ }^{15,16}$ Matthew Weiss, ${ }^{4,17}$ Maureen Meade, ${ }^{18}$ \\ Pierre Marsolais, ${ }^{19}$ Sam Shemie, ${ }^{20}$ Sanabelle Zaabat, ${ }^{21}$ Sonny Dhanani, ${ }^{22}$ \\ Simon C Kitto, ${ }^{23}$ Michaël Chassé, ${ }^{3,15,16}$ on behalf of the Canadian Donation and \\ Transplantation Research Program and the Canadian Critical Care Trials Group
}

To cite: Crawshaw J, Presseau J, van Allen Z, et al. Exploring the experiences and perspectives of substitute decision-makers involved in decisions about deceased organ donation: a qualitative study protocol. BMJ Open 2019;9:e034594. doi:10.1136/ bmjopen-2019-034594

- Prepublication history for this paper is available online. To view these files, please visit the journal online (http://dx.doi. org/10.1136/bmjopen-2019034594).

Received 26 September 2019 Revised 26 November 2019 Accepted 05 December 2019

Check for updates

(C) Author(s) (or their employer(s)) 2019. Re-use permitted under CC BY-NC. No commercial re-use. See rights and permissions. Published by BMJ.

For numbered affiliations see end of article.

Correspondence to Dr Michaël Chassé; michael.chasse@umontreal.ca

\section{ABSTRACT}

Introduction In Canada, deceased organ donation provides over $80 \%$ of transplanted organs. At the time of death, families, friends or others assume responsibility as substitute decision-makers (SDMs) to consent to organ donation. Despite their central role in this process, little is known about what barriers, enablers and beliefs influence decision-making among SDMs. This study aims to explore the experiences and perspectives of SDMs involved in making decisions around the withdrawal of life-sustaining therapies, end-of-life care and deceased organ donation.

Methods and analysis SDMs of 60 patients admitted to intensive care units will be enrolled for this study. Ten hospitals across five provinces in Canada in a prospective multicentre qualitative cohort study. We will conduct semistructured telephone interviews in English or French with SDMs between 6 and 8 weeks after the patient's death. Our sampling frame will stratify SDMs into three groups: SDMs who were not approached for organ donation; SDMs who were approached and consented to donate and SDMs who were approached but did not consent to donate. We will use two complementary theoretical frameworks - the CommonSense Self-Regulation Model and the Theoretical Domains Framework - to inform our interview guide. Interview data will be analysed using deductive directed content analysis and inductive thematic analysis.

Ethics and dissemination This study has been approved by the Centre Hospitalier de l'Université de Montréal Research Ethics Board. The findings from this study will help identify key factors affecting substitute decisionmaking in deceased organ donation, reasons for nonconsent and barriers to achieve congruency between SDM and patient wishes. Ultimately, these data will contribute to the development and evaluation of tools and training for

\section{Strengths and limitations of this study}

- Our study will explore diverse substitute decisionmaker (SDM) perspectives across five Canadian provinces which have different organ donation systems.

- We propose an innovative sampling strategy that will directly and prospectively identify SDMs to minimise the risk of selection bias.

- We will draw on two complementary theoretical frameworks to understand decision-making processes in end-of-life contexts.

- Our study has been endorsed by leading Canadian networks in organ donation and transplantation and critical care

- Our findings may not be generalisable to intensive care units in jurisdictions we will not sample and for non-English or non-French speakers.

healthcare providers to support SDMs in making decisions about organ donation.

Trial registration number NCT03850847.

\section{INTRODUCTION}

The Canadian organ transplantation system relies on altruistic deceased organ donation. In Canada, over $80 \%$ of organs are transplanted from deceased donors. ${ }^{12}$ At the end of 2017, over 4300 Canadians were awaiting solid organ transplantation. Of these, 242 died while on the transplant waiting list. $^{12}$ Strategies to improve donation rates are continuously sought by the medical 
community, policy makers, transplantation communities and citizens. ${ }^{3}$

Consent for organ donation in Canada requires direct discussions between trained donation experts and substitute decision-makers (SDMs) (eg, families, friends or significant persons in the patient's life), who may or may not be aware of the patients' premorbid wishes, whether these wishes were formally registered or not (eg, online or through health card or drivers' license applications). As organ donation preferences represent personal values, some SDMs may refuse to engage in these discussions. Furthermore, data suggest substantial mismatch between individuals' stated wishes and decisions made by SDMs. ${ }^{4}$ While $90 \%$ of Canadians surveyed support organ donation, ${ }^{1}$ over one-third of Canadian families decline organ donation when approached. In Ontario, one-fifth of SDMs of registered donors ultimately declined organ donation. ${ }^{5}$

Despite the central role of the substitute decisionmaking process, our understanding about the factors influencing the decision around deceased organ donation is restricted to few studies, most of which have been conducted outside of Canada. ${ }^{6}$ A qualitative study involving $27 \mathrm{SDMs}$ in Ontario who had been involved in discussions regarding consent for organ donation found that although participants reported empathetic care from hospital staff, procedural (eg, communication) and situational (eg, a proper setting for family meetings) factors left family members 'troubled by unanswered questions'. ' Current research is insufficient to guide personalised strategies for supporting SDMs, ${ }^{8}$ which remain largely ad hoc rather than informed by evidence. Therefore, understanding which factors best account for SDM decisions and how their views affect that process is of utmost importance. Most studies to date have not directly involved SDMs and have been limited by inappropriate sampling such as retrospective selection, ${ }^{7} 10$ which is associated with selection bias. Others have failed to use structured and validated models or frameworks in their design and analysis. 61112 As a result, there is substantial opportunity to ensure that SDMs are supported to make decisions that are as informed as possible in these challenging, emotionladen circumstances where withdrawal of life-sustaining therapies, end-of-life care and organ donation need to be discussed.

To address these gaps, we seek to gain a better understanding of the views and experiences of SDMs regarding the process of consent to organ donation. We therefore propose a prospective qualitative study of SDM views and experiences to support and improve the decision-making process regarding deceased organ donation. Consistent with a previously reported approach, ${ }^{13}$ we will draw on two complementary theoretical frameworks, Leventhal's Common Sense Self-Regulation Model (CSSRM) ${ }^{14}$ and the Theoretical Domains Framework (TDF), ${ }^{15}$ that to our knowledge, have yet to be employed in the context of organ donation.

\section{AIMS}

Our aim is to investigate the experience and perspectives of SDMs involved in decisions around the withdrawal of life-sustaining therapies, end-of-life care and deceased organ donation.

\section{METHODS AND ANALYSIS \\ Study design}

We will conduct a prospective, multicentre, qualitative cohort study including semistructured telephone interviews with the SDMs of potential organ donors. This study constitutes step 1 of the Understanding Decision Making in the Intensive Care Unit: a National Study.

\section{Setting and context}

Ten academic hospital centres in Canada, each active in organ donation, will participate. This study will be conducted in collaboration with the Canadian Critical Care Trials Group (CCCTG) and the Canadian Donation and Transplant Research Program (CDTRP).

\section{Sample and recruitment}

At each participating site, research coordinators with experience working with critically ill populations will prospectively identify consecutive patients admitted in the intensive care unit (ICU) for whom withdrawal of life-sustaining therapies and/or organ donation has been discussed or are about to be discussed. Research coordinators will then identify and approach the $\operatorname{SDM}(\mathrm{s})$ of these patients through discussion with ICU physicians and the nursing team. At this point, SDMs will be provided a letter of information including detailed information about the study. We will include at least one SDM per patient, however, the opportunity to participate will be offered to all other interested SDMs. At the initial contact, research coordinators will simply solicit preconsent to be contacted by phone between 6 and 8 weeks after the patient's death at a time convenient to the SDM.

We will interview SDMs from up to 60 patients using a purposive sampling approach to obtain a variety of perspectives. As such, our sampling frame will equally stratify interviews with SDMs who were: not approached about organ donation (NA group), approached and consented to donate (AC group) and approached but did not consent (ANC group).

\section{Theoretical framework}

We will first draw on the $\mathrm{CSSRM}^{14}$ of health and illness to explore how SDMs understood and conceptualised the illness/injury that caused the death of the patient. The CSSRM proposes that individuals hold common sense beliefs about a particular health threat (eg, illness / injury), and these beliefs allow them to make sense of symptoms and illness experiences. These beliefs, or 'illness representations', vary in nature and scope between and within individuals over time and comprise five inter-related constructs: identity; cause; timeline; 
consequences; cure/control. The CSSRM considers these illness representations alongside emotional reactions (eg, fear, worry, guilt, sadness) and proposes a model for how individuals cope with these representations and their emotions in response. We will explore how SDMs labelled the illness/injury in their own words (identity), what they believed led to the illness/injury (cause), how long they believed the patient had left to live following the illness/injury (timeline), how their views changed over time about the consequences of the illness/injury (consequences) and their beliefs about curability and reversibility of the illness/injury (cure/control). We will also explore the role of their emotional reactions during this process.

We will then draw on the Theoretical Domains Framework (TDF $)^{15}$ to explore the barriers and enablers that influenced or may have influenced the decisions made by SDMs about the withdrawal of life-sustaining therapies, end-of-life care and the organ donation process. The TDF summarises constructs from predominant theories of behaviour and behaviour change into 14 distinct theoretical domains each representing key factors that determine behaviour (knowledge, skills, social/professional role and identity, beliefs about capabilities, beliefs about consequences, optimism, reinforcement, intentions, goals, memory/ attention/decision processes, environmental context and resources, social influences, emotion, behavioural regulation (see Cane and colleagues ${ }^{16}$ for definitions). The TDF provides a basis for understanding a broad set of factors that influence the decision to consent to donate or not and has been used as a basis to identify potential barriers and enablers to behaviours in other settings. ${ }^{16-19}$ Although the TDF has been used to explore the views of ICU staff and organ donor coordinators involved in deceased organ donation, ${ }^{19}$ as far as we are aware, it has yet to be used among SDMs involved in organ donation.

\section{Interviews}

Interview guide

Interviews will be performed in a semistructured format using an interview guide, which will include open-ended questions based on the constructs and domains from the CSSRM and TDF, respectively. The questions will cover the experiences and perspectives of SDMs around the withdrawal of life-sustaining therapies, end-of-life care and the organ donation process. We will explore factors that influenced their decision to consent to donate or not (or that would have influenced their decision for those not approached), and how such factors may have changed over the course of making this decision and since the decision (or since the event for those not approached). The interview guide will be developed collaboratively with our patient-partners and will be pilot tested internally within the research team and externally with patient-partners from the investigators' research network for perceived clarity, brevity and sensitivity.

\section{Interviewer training}

Interviews will be conducted in English or French by a multidisciplinary team of researchers and clinicians trained in qualitative research methodology and experienced with critically ill populations. One of our patientpartners, with lived experience of deceased organ donation, will be an interviewer. Each member of the interview team will be trained to: familiarise themselves with the interview guide; undertake practice interviews using training vignettes and undertake practice interviews with SDMs with lived experience of deceased organ donation who have volunteered to collaborate in this phase of the study. Continued refinement of the interview guide will take place during this training process until a final version is agreed on prior to recruitment of study participants.

\section{Data collection}

We will collect patient demographic and clinical data during the patients' ICU stay (age, sex, type of injury/ illness, cause of death, organ donation status). We will collect SDM demographic data (age, gender, religion, ethnicity) and obtain audiorecorded informed verbal consent from SDMs at the time of the scheduled interviews. All interviews will be conducted between 6 and 8 weeks after the patient's death to minimise family burden, optimise research consent rates and avoid influencing the donation process. This approach was successful in previous studies involving bereaving families ${ }^{20-23}$ and is supported by our patient partners.

The coordinating centre will post a reminder letter to SDMs within a 4-week period after the patient's death, suggesting a date and time for the first phone call with the interviewer (scheduled between 6 and 8 weeks after the patient's death). If the SDM cannot be reached at the first call, interviewers will be instructed to make up to five more call attempts to schedule the interview over the next 3 -week period. If the SDM is contacted but is not available to be interviewed at the time of the first phone call, they will be able to postpone/reschedule the interview up to three times, until a 6-month cut-off point is reached (eg, 6 months since the death of the patient). SDMs who cannot be contacted by phone within the 6-month cut-off point will be excluded.

Interviews will continue until thematic data saturation is achieved (see Data analysis section). Thematic data saturation will be determined using a formal stopping rule $^{24}$ : when no new themes emerge within TDF domains or CSSRM constructs in 3 consecutive interviews after at least 10 interviews have been conducted per group. In previous theory-based interview studies, ${ }^{25}{ }^{26}$ saturation was typically achieved within 15-25 interviews per group. Thus, targeting a total of 60 patients (20 per group) should be sufficient to achieve data saturation. Interviews will be transcribed verbatim. French interviews will be translated by trained professionals to allow data analysis to be conducted in English. 


\section{Data analysis}

Qualitative analyses will include both deductive directed content analysis ${ }^{27}$ and inductive thematic analysis. ${ }^{28}$ The theoretical basis - the CSSRM and the TDF-of the interview guide will inform the directed content analysis. A coding manual will describe each CSSRM construct and TDF domain and provide flexibility to code emerging themes. Two researchers will independently code anonymised transcripts using NVivo qualitative data analysis software (QSR International Pty V.11, 2015). Respondent utterances will be coded to a CSSRM construct or TDF domain. Coders will begin by coding one transcript selected at random from the first 10 interviews conducted in each group (NA, AC and ANC) and then meet to compare coding and identify discrepancies. Discrepancies will be discussed with a third member until a consensus is reached and the final decision will be added to the coding manual. Independent double coding will then proceed in blocks of five transcripts, followed by discrepancy discussions and necessary adjustments to the coding manual. Krippendorff's alpha ${ }^{29}$ will be used to assess inter-rater agreement within each block of five and overall. We will assess the relevance of constructs/ domains based on three factors, as per recommendations in the literature ${ }^{17}$ : frequency of shared beliefs across respondents, incidence of conflicting beliefs and particular emphasis of the importance of a given belief in every respondent experience. ${ }^{18}$ Following directed content analysis, our coders will conduct thematic analysis of content within constructs/domains which will involve: searching for themes, reviewing themes and defining and naming themes. ${ }^{28}$ Data will be reported in accordance with the Consolidated Criteria for Reporting Qualitative Research guidelines. $^{30}$

\section{Patient and public involvement}

Input from patient-partners with lived experience of deceased organ donation will help to shape the design and delivery of this study. One of our interviewers (a coapplicant on our study grant) is a patient-partner with lived experience of deceased organ donation. Our interview guide will be developed collaboratively with our patient-partners and will be pretested for perceived clarity, brevity and sensitivity by the research team and patient-partners (see Interview guide section). The interview team will undertake practice interviewers with patient-partners from non-profit organisations (eg, Chain of Life) to ensure they are competent using the interview guide (see Ethical/safety considerations section). Feedback from patient-partners about the practice interviews will be collated and discussed among the research team.

\section{ETHICS AND DISSEMINATION}

\section{Ethical/safety considerations}

At the time of patient identification, only preconsent to be contacted by phone (6-8 weeks later) will be obtained from the patient's SDMs. Despite seemingly minimal commitment at this point, we are aware that even asking potentially distressed and grieving individuals to consider research participation must be handled sensitively. ${ }^{31} 32$ Our research coordinators, who have experience working with critically ill populations, will approach SDMs in a dignified and compassionate manner and will be attuned to recognise any negative reactions towards being approached about research participation. Other studies have used a similar approach to consent at the time of bereavement, ${ }^{33}$ and there is some evidence that participating in research may benefit those suffering bereavement. ${ }^{34}$

Informed consent (verbal, recorded) and data collection will take place $6-8$ weeks after the patient's death. Acknowledging that the ideal timing for this contact may vary among SDMs, we will offer the opportunity to postpone/reschedule the interview up to three times (see Data collection section). We will contact SDMs at a time when they may still be distressed about revisiting a painful memory. Interviews will be conducted by a multidisciplinary team of researchers and clinicians trained in qualitative research methodology and experienced with critically ill populations. Interviewers will undergo internal practice within the research team and with patient-partners from non-profit organisations (eg, Chain of Life). Interviewers will also attend a 1 day workshop run by the Distress Center of Ottawa and Region on how to deal with challenging situations by phone (eg, active listening, communication and empathic assertiveness).

If the interviews exacerbate emotional distress (eg, signs of anxiety, continued crying or an inability to continue the conversation), the interviewers will be instructed to gently interrupt the interview and ask the interviewee if they wish to be referred to medical staff via the site research coordinator who will be immediately notified. The interviewee will also be directed to the Distress Center of Ottawa and Region's 24/7 Distress Line which offers confidential support for those experience difficulties in their life (https://www.dcottawa.on.ca/).

We will also have escalation (eg, confidential helpline to at each site) and debrief (eg, postinterview reflection forms, regular debrief meetings with the interview team) processes in place to protect the well-being of our interview team in case they are emotionally affected by anything discussed during the interviews and to identify potential researcher burnout. ${ }^{35}$

All information collected during the study will be held in strict confidence to the extent provided by law. Each patient record and each SDM record will be pseudonymised with a unique study ID and will be kept by the research coordinator at each site. Study data will be collected on paper and electronic case report forms and will be stored for at least 10 years by the principal investigator.

\section{Impact and dissemination}

The findings from this study will help to identify key factors affecting substitute decision-making in deceased organ donation, reasons for non-consent and barriers to achieve congruency between SDM and patient wishes. 
Moreover, these data will help us better understand the decision-making process in the context of end-of-life care for critically ill patients in Canada. Findings from this study will inform step 2 of the Understanding Decision Making in the Intensive Care Unit: a National Study, which will involve the design of a national survey among SDMs exploring key factors influencing decision-making regarding deceased organ donation. We also plan to convene a 1-day workshop comprising investigators, stakeholders, knowledge-users and patient-partners to analyse and interpret the main results of the study, identify key elements and strategies for dissemination and help plan for the next steps required to develop an implementation strategy. Ultimately, these data will contribute to the development and evaluation of tools and training for healthcare providers to optimise approaches to support SDMs in the context of organ donation.

This study will be conducted in collaboration with the CCCTG and CDTRP, whose members will help to guide the analyses, interpretation and dissemination of our findings through feedback at scientific meetings. We have also engaged knowledge translation leaders for organ donation at the Canadian Blood Services, Transplant Québec and Trillium Gift of Life to ensure uptake of the results by relevant stakeholders.

\section{Study status}

We started enrolment in August 2019, and we expect to complete the study within 12 months (6 months recruitment; 9 months concurrent data analysis).

\section{Author affiliations}

${ }^{1}$ Clinical Epidemiology Program, Ottawa Hospital Research Institute, Ottawa, Ontario, Canada

${ }^{2}$ School of Epidemiology and Public Health, University of Ottawa, Ottawa, Ontario,

Canada

${ }^{3}$ Innovation Hub, Centre de Recherche du CHUM, Montréal, Québec, Canada

${ }^{4}$ Canadian Donation and Transplant Research Program, Edmonton, Alberta, Canada

${ }^{5}$ Medicine (Critical Care), University of Ottawa, Ottawa, Ontario, Canada

${ }^{6}$ Population Health and Optimal Health Practices Unit (Trauma-Emergency-Critical

Care Medicine), CHU de Quebec-Universite Laval, Quebec City, Québec, Canada

${ }^{7}$ Department of Anesthesiology and Critical Care, Division of Critical Care Medicine, Université Laval, Quebec City, Québec, Canada

${ }^{8}$ Critical Care Medicine, Ottawa Hospital, Ottawa, Ontario, Canada

${ }^{9}$ Medicine (Critical Care), Schulich School of Medicine and Dentistry, London,

Ontario, Canada

${ }^{10}$ Anesthesiology, Universite de Sherbrooke Faculte de medecine et des sciences de la sante, Sherbrooke, Quebec, Canada

${ }^{11}$ Centre de recherche du CHUS, Sherbrooke, Quebec, Canada

${ }^{12}$ University of Ottawa Faculty of Medicine, Ottawa, Ontario, Canada

${ }^{13}$ Medicine, London Health Sciences Centre, London, Ontario, Canada

${ }^{14}$ Critical Care, St Michael's Hospital, Toronto, Ontario, Canada

${ }^{15}$ Medicine (Critical Care), Centre Hospitalier de L'Universite de Montreal, Montréal,

Québec, Canada

${ }^{16}$ Medicine (Critical Care), Université de Montréal, Montreal, Québec, Canada

${ }^{17}$ Trauma-Emergency-Critical Care Medicine, Université Laval Faculté de médecine,

Quebec City, Quebec, Canada

${ }^{18}$ Medicine (Critical Care), McMaster University, Hamilton, Ontario, Canada

${ }^{19}$ Medicine, Hôpital du Sacré-Coeur de Montréal, Montreal, Québec, Canada

${ }^{20}$ Critical Care, McGill University, Montreal, Québec, Canada

${ }^{21}$ Medicine, Université de Montréal, Montréal, Québec, Canada

${ }^{22}$ Critical Care, CHEO, Ottawa, Ontario, Canada

${ }^{23}$ Innovation in Medical Education, University of Ottawa, Ottawa, Ontario, Canada
Twitter Jacob Crawshaw @JakeECrawshaw, lan Ball @Ball and Simon C Kitto @ DrSimonKitto

Acknowledgements The authors thank the support from the CCCTG and its Grant and Manuscript Review Committee (Erick Duan) for helpful comments on the manuscript. We would also like to thank all the patient-partners who have helped shape the design and delivery of this study.

Collaborators The Canadian Critical Care Trials Group and the Canadian Donation and Transplantation Research Program

Contributors All authors (JC, JP, ZvA, LPC, KJ, SE, DAF, FL, AFT, AJS, CM, FD, AHL, GK, IB, JB, KEAB, MCF, MW, MM, PM, SS, SZ, SD, SCK, MC) participated in conceiving this study. The conduct of the study will be led by MC, JP and KJ. All authors provided input into the protocol, critical feedback on the manuscript, and approved the final manuscript.

Funding This study is supported by the Canadian Institute of Health Research, grant number PJT-155997. MC, FL, FDA and MCF are recipients of a salary support award from the Fonds de Recherche Québec - Santé (FRQS). AFT is the chair holder of the Canada Research Chair in Critical Care Neurology and Trauma.

Competing interests None declared.

Patient consent for publication Not required.

Ethics approval A prospective multicentre qualitative cohort study has been approved by the Centre Hospitalier de l'Université de Montréal Research Ethics Board (MP-02-2019-7792). Provincial and local ethics approval will also be obtained from each participating site.

Provenance and peer review Not commissioned; externally peer reviewed.

Open access This is an open access article distributed in accordance with the Creative Commons Attribution Non Commercial (CC BY-NC 4.0) license, which permits others to distribute, remix, adapt, build upon this work non-commercially, and license their derivative works on different terms, provided the original work is properly cited, appropriate credit is given, any changes made indicated, and the use is non-commercial. See: http://creativecommons.org/licenses/by-nc/4.0/.

\section{ORCID iDs}

Jacob Crawshaw http://orcid.org/0000-0002-6302-1496

Alexis F Turgeon http://orcid.org/0000-0001-5675-8791

\section{REFERENCES}

1 Canadian Blood Services. Organ donation and transplantation in Canada: system progress report - 2017 update. Canadian Blood Services, 2018.

2 Canadian Institute for Health Information. Annual statistics on organ replacement in Canada: dialysis, transplantation and donation, 2008 to 2017. Canadian Institute for Health Information, 2018.

$3 \mathrm{Li}$ AH, Naylor KL, Garg AX, et al. Interventions for increasing solid organ donor registration. Cochrane Database Syst Rev 2013:CD010829.

4 Anthony SJ, Toews M, Caulfield T, et al. Family veto in organ donation in Canada: framing within English-language newspaper articles. CMAJ Open 2017;5:E768-72.

5 Toews M, Caulfield T. Evaluating the "family veto" of consent for organ donation. Can Med Assoc J 2016;188:E436-7.

6 Walker W, Broderick A, Sque M. Factors influencing bereaved families' decisions about organ donation: an integrative literature review. West J Nurs Res 2013;35:1339-59.

7 Sarti AJ, Sutherland S, Healey A, et al. A multicenter qualitative investigation of the experiences and perspectives of substitute decision makers who underwent organ donation decisions. Prog Transplant 2018;28:343-8.

8 Shah SK, Kasper K, Miller FG. A narrative review of the empirical evidence on public attitudes on brain death and vital organ transplantation: the need for better data to inform policy. $J$ Med Ethics 2015;41:291-6.

9 Marck CH, Neate SL, Skinner MR, et al. Factors relating to consent for organ donation: prospective data on potential organ donors. Intern Med J 2015;45:40-7.

10 Neate SL, Marck CH, Skinner M, et al. Understanding Australian families' organ donation decisions. Anaesth Intensive Care 2015;43:42-50.

11 Sque M, Walker W, Long-Sutehall T, et al. Bereaved donor families' experiences of organ and tissue donation, and perceived influences on their decision making. J Crit Care 2018;45:82-9. 
12 Walker W, Sque M. Balancing hope and despair at the end of life: the contribution of organ and tissue donation. J Crit Care 2016;32:73-8.

13 Langston AL, Johnston M, Robertson C, et al. Protocol for stage 1 of the gap study (genetic testing acceptability for Paget's disease of bone): an interview study about genetic testing and preventive treatment: would relatives of people with Paget's disease want testing and treatment if they were available? BMC Health Serv Res 2006;6:71.

14 Leventhal H, Diefenbach M, Leventhal EA. Illness cognition: using common sense to understand treatment adherence and affect cognition interactions. Cognit Ther Res 1992;16:143-63.

15 Michie S, Johnston M, Abraham C, et al. Making psychological theory useful for implementing evidence based practice: a consensus approach. Qual Saf Health Care 2005;14:26-33.

16 Cane J, O'Connor D, Michie S. Validation of the theoretical domains framework for use in behaviour change and implementation research. Implement Sci 2012;7.

17 Atkins L, Francis J, Islam R, et al. A guide to using the theoretical domains framework of behaviour change to investigate implementation problems. Implement Sci 2017;12:77.

18 Patey AM, Islam R, Francis JJ, et al. Anesthesiologists' and surgeons' perceptions about routine pre-operative testing in low-risk patients: application of the theoretical domains framework (TDF) to identify factors that influence physicians' decisions to order preoperative tests. Implement Sci 2012;7:52.

19 Squires JE, Graham N, Coughlin M, et al. Barriers and enablers to organ donation after circulatory determination of death: a qualitative study exploring the beliefs of frontline intensive care unit professionals and organ donor coordinators. Transplant Direct 2018;4:e368.

20 Downar J, Barua R, Sinuff T. The desirability of an intensive care unit (ICU) clinician-led bereavement screening and support program for family members of ICU decedents (ICU Bereave). J Crit Care 2014;29:311.e9-6.

21 Cleiren MPHD, Zoelen AJV. Post-Mortem organ donation and grief: a study of consent, refusal and well-being in bereavement. Death Stud 2002;26:837-49.

22 de Groot J, van Hoek M, Hoedemaekers C, et al. Request for organ donation without donor registration: a qualitative study of the perspectives of bereaved relatives. BMC Med Ethics 2016;17:38.
23 van Beinum A, Hornby L, Dhanani S, et al. Feasibility of conducting prospective observational research on critically ill, dying patients in the intensive care unit. J Med Ethics 2017;43:47-51.

24 Francis JJ, Johnston M, Robertson C, et al. What is an adequate sample size? Operationalising data saturation for theory-based interview studies. Psychol Health 2010;25:1229-45.

25 Presseau J, Mutsaers B, Al-Jaishi AA et al. Barriers and facilitators to healthcare professional behaviour change in clinical trials using the theoretical domains framework: a case study of a trial of individualized temperature-reduced haemodialysis. Trials 2017;18:227.

26 Presseau J, Schwalm JD, Grimshaw JM, et al. Identifying determinants of medication adherence following myocardial infarction using the theoretical domains framework and the health action process approach. Psychol Health 2017;32:1176-94.

27 Hsieh H-F, Shannon SE. Three approaches to qualitative content analysis. Qual Health Res 2005;15:1277-88.

28 Braun V, Clarke V. Using thematic analysis in psychology. Qual Res Psychol 2006;3:77-101.

29 Krippendorff K. Reliability in content analysis. Hum Commun Res 2004;30:411-33.

30 Tong A, Sainsbury P, Craig J. Consolidated criteria for reporting qualitative research (COREQ): a 32-item checklist for interviews and focus groups. Int J Qual Health Care 2007;19:349-57.

31 Azoulay E, Chaize M, Kentish-Barnes N. Involvement of ICU families in decisions: fine-tuning the partnership. Ann Intensive Care 2014;4:37.

32 Kentish-Barnes N, McAdam JL, Kouki S, et al. Research participation for bereaved family members: experience and insights from a qualitative study. Crit Care Med 2015;43:1839-45.

33 Kentish-Barnes N, Chaize M, Seegers V, et al. Complicated grief after death of a relative in the intensive care unit. Eur Respir $J$ 2015;45:1341-52.

34 Sque M, Walker W, Long-Sutehall T. Research with bereaved families: a framework for ethical decision-making. Nurs Ethics 2014;21:946-55

35 Butler AE, Copnell B, Hall H. Researching people who are bereaved: managing risks to participants and researchers. Nurs Ethics 2019;26:224-34. 